\title{
Socio-Cultural Barriers Influencing Utilization of Sexual and Reproductive Health (SRH) Information and Services among Adolescents and Youth 10 - 24 Years in Pastoral Communities in Kenya
}

\author{
Joyce Wangui Kinaro ${ }^{*}$, Gilbert Wangalwa², Sarah Karanja ${ }^{3}$, Ben Adika ${ }^{4}$, \\ Catherine Lengewa ${ }^{4}$, Patric Masitsa ${ }^{5}$ \\ ${ }^{1}$ KnowSolve Consulting Ltd., Nairobi, Kenya \\ ${ }^{2}$ Afya Timiza, Amref Health Africa in Kenya, Nairobi, Kenya \\ ${ }^{3}$ Amref Health Africa in Kenya, Nairobi, Kenya \\ ${ }^{4}$ Centre for Behavior Change Communication, Nairobi, Kenya \\ ${ }^{5}$ Independent Consultant, Nairobi, Kenya \\ Email: ${ }^{*}$ mailto:jwkinaro@gmail.com
}

How to cite this paper: Kinaro, J.W., Wangalwa, G., Karanja, S., Adika, B., Lengewa, C. and Masitsa, P. (2019) Socio-Cultural Barriers Influencing Utilization of Sexual and Reproductive Health (SRH) Information and Services among Adolescents and Youth 10 24 Years in Pastoral Communities in Kenya. Advances in Sexual Medicine, 9, 1-16. https://doi.org/10.4236/asm.2019.91001

Received: September 25, 2018 Accepted: December 22, 2018 Published: December 25, 2018

Copyright $\odot 2019$ by authors and Scientific Research Publishing Inc. This work is licensed under the Creative Commons Attribution International License (CC BY 4.0).

http://creativecommons.org/licenses/by/4.0/

\begin{abstract}
Background: As in other developing countries, sexual and reproductive ill-health continues to mostly affect adolescents and youths. Samburu and Turkana counties in Kenya have some of the highest levels of total fertility rates (TFR) at 6.3 and 6.9 respectively placing them well above the national TFR of 3.9. Establishing factors that influence utilization of SRH services among adolescent and youth aged $10-24$ years is critical in developing an effective program. Method: We used primary data from qualitative and purposeful study design. Data collection used Focus group discussions (FGD), In-depth interviews (IDIs) and Key informant interviews (IDIs). The target groups were adolescents and youth aged $10-24$ years, health care providers, community health volunteers (CHVs), chemist assistants, parents of adolescents and youth, teachers, spiritual leaders and traditional activists. Findings and Conclusion: Socio-cultural factors were found to influence utilization of SRH services and information. Early marriage, being youth, male only decisions on sexuality matters and fear of family contribute to unprotected sex while myths and misconceptions on contraceptives affected utilization. The findings revealed that youth needs to know sources, how contraceptives work and how to use them. The findings suggest capacity building of health care
\end{abstract}


providers, $\mathrm{CHVs}$, teachers, parents and community leaders on adolescence, sexuality needs of adolescents and disadvantages of female genital mutilation (FGM) including early marriage.

\section{Keywords}

Adolescents and Youth, Socio-Cultural, Sexuality Information, Service

Utilization, Sexual and Reproductive Health

\section{Introduction}

Despite the commitment at the International Conference for Population and Development (ICPD), sexual and reproductive ill-health continues to mostly affect adolescents and youths worldwide [1]. The poor reproductive health ( $\mathrm{RH})$ outcomes among adolescents and youths have been attributed to low use of contraception or methods to prevent sexually transmitted infections or human immunodeficiency virus (STI/HIV) infection, physiological immaturity, inadequate use of reproductive health care services, and poor socio-economic conditions [2]. Despite the risks associated with early pregnancies, studies indicate sexually active 15 - 19 years old adolescents rarely use contraceptives [3]. In Kenya, the percentage of women who have begun childbearing increases rapidly with age, from about 3 percent among women aged 15 years to 40 percent among women aged 19 years [4]. Information on sexuality and reproductive health and sources of services is therefore important and paramount.

According to the 2009 Kenya Population and Housing Census (KPHC), Kenya has a broad based (pyramid shaped) population structure [5]. The structure shows that 63 percent of the population is below 25 years and 32 percent is aged between 10 - 24 years [5]. The youthful age structure presents socio-economic and health services provision challenges for the country as majority of the youth at this age is still in school and dependent or not gainfully employed. Despite the National Adolescent Sexual and Reproductive Health Policy (2015) providing guidance towards responding to adolescents SRH needs, inadequate knowledge of SRH issues continues to expose adolescents to risky sexual behavior [6]. The policy recommends the need for an integrated approach providing for mitigation of risk factors and prevention of SRH challenges [6]. It is critical for SRH programs to use the policy to guide in making services and information more accessible to adolescents and youth.

According to the social and cognitive theory, behavior is a product of perceptions while perception is a function of different factors that include socio-cultural influences [7]. Perception formation theory indicates that behavior is influenced by information adolescents receive from the family, school and the media [7] [8]. According to Hulton [9], socio-cultural factors influence how decisions are made and the confidence to use contraceptives and other RH services [10]. Both theoretical and empirical literatures show that the influence of family or com- 
munity on adolescent behavior represents the central ways in which parents influence healthy adolescent development [11] [12]. Undesired perceptions could be as a result of myths and misconceptions acquired through socialization and interactions in the community [12] [13]. Evidence has shown that cultural values and beliefs of health care providers play a critical role in deciding whether or not to provide contraception to adolescents [13]. Even when adolescents have desired perceptions and are ready to utilize a service, socio-cultural values by health providers may hinder access to reproductive health services by unmarried youth [14] [15]. It has been documented that health care providers' perception that unmarried youth should not be given contraceptives and should at least give birth first to prove fertility [16] may hinder SRH services provision. It is important therefore, to address health care providers' perceptions brought about by their cultural orientation that would hinder utilization of reproductive health services by the youth.

Socio-cultural factors have been found to influence gender relationships in the utilization of SRH services. Studies have revealed substantial gender inequities in power relations in sexuality issues that illustrates the role of gender as a determinant of sexuality and risky behavior in society [17]. It has been reported women aged 15 - 24 years old are twice more likely to be infected with HIV than boys of the same age [18]. Women have been found to do very little to influence condom use by their partners to protect themselves from HIV due to the perception that condom use implied infidelity and they could not space or stop having children without their male partner's permission [19] [20]. Partner communication has also been found to be significant in contraceptive use among adolescents aged between 15 - 19 years old [12]. Studies have also shown that programs that emphasized specific skills, such as partner communication on negotiation skills tended to increase utilization of SRH services than programs that stress general knowledge [11] [21]. For adolescents to adopt contraceptive use and other SRH information and services, they need to overcome perceptions and misconceptions influenced by socio-cultural barriers. It is critical therefore to integrate the community leaders in the implementation of programs that address utilization of SRH services and information using content relevant to the local culture. This paper therefore seeks to discuss socio-cultural barriers that influence utilization of SRH information and services among adolescents and youth 10 - 24 years in the pastoral communities of Samburu and Turkana Counties in Kenya.

\section{Methods}

\subsection{Study Setting}

The study was carried out in Samburu and Turkana counties that have some of the highest levels of total fertility rates (TFR) in Kenya. The TFR in Samburu and Turkana is estimated at 6.3 and 6.9 respectively placing them well above the national TFR of 3.9 [4]. Whereas Samburu shares its borders with four other 
counties; Marsabit to the north and northeast, Isiolo to the east, Turkana to the west and northwest, and Laikipia and Baringo to the southwest. The county covers an area of $21,022.27 \mathrm{sq} \mathrm{km}$ with a population of about 224,000 people according to 2009 National census [22]. The Samburu's rely on sheep, goats, cattle and camels as their source of livelihood. Majority of people living in Samburu County adhere to traditional beliefs, although some Samburus have converted to Christianity [22].

Turkana on the other hand shares its borders with not only Samburu, but with other four counties namely Marsabit to the east, Samburu to the south-east, Baringo and West Pokot to the south-west. The county also borders with South Sudan to the north, Uganda to the west and Ethiopia to the north-east. It has a population of about 855,399 people according to 2009 National census [22] of whom majority are youthful. The Turkana are nomadic pastoralists who mainly keep cattle, donkeys, camels and goats. The animals are their main source of food and wealth. Close to $95 \%$ of the people living in Turkana County adheres to traditional beliefs while $5 \%-10 \%$ of the residents practice Christianity [22].

\subsection{Study Design}

This paper draws on primary data collected using qualitative and purposeful study design to gather in-depth information underlying the issues that influence adolescents and youth to access and utilize SRH services and information. The study was carried out between April to June 2017 in two sub-Counties in Samburu County and three sub-Counties in Turkana. The participants were selected from Samburu Central and Samburu East sub-Counties of Samburu County and Kibish, Loima and Turkana South sub-Counties of Turkana.

An inclusive criteria for the sample was adolescents and youth aged between 10 - 24 years. A purposive sampling technique was used to select participants for the study among them: adolescents and youth aged $10-14,15-19$ and $20-24$ years old, residence (urban and rural), gender (male and female), marital status (married and unmarried) and schooling (out and in school). Parents of the youth, health care providers, community health volunteers, religious leaders and traditional activists ${ }^{1}$ were also identified as Key informants and for in depth interviews.

\subsection{Data Collection}

Data collection was carried out by research assistants who were selected from the community and trained. Data collectors had experience working in the community in similar activities and ability to communicate in the local language and record data in English. Adolescents and youth 10 - 24 years participated in focus group discussions (FGDs) and in-depth interviews (IDIs). Each FGD had between 8 - 12 participants. All discussions in the study had one interviewer and one note taker. The interviews lasted one hour and were tape recorded. We ${ }^{1}$ Traditional activists were opinion leaders in the community. 
conducted 40 FGDs in several sessions with a mixed gender youth who comprised of 20 males and 20 females. The 50 IDIs conducted among the youth comprised of 29 males and 21 females. We conducted 51 key informant interviews (KIIs) that included 18 males and 33 females. Participants for KIIs were 11 healthcare providers, 14 community health volunteers ${ }^{2}$, five chemist assistants, 9 parents of adolescents and youth, eight teachers, two spiritual leaders and activists as shown in Table 1.

The study was carried out among pastoral communities whose homes were spread far apart from each other. This prevented interviews from taking place in respondent's homes. To overcome this challenge, respondents were notified beforehand and informed to present themselves at a predetermined venue. At each venue, participants were interviewed in privacy. A refund of travel costs of the participants was done after the interviews.

\subsection{Ethical Consideration}

Study approvals were obtained from Amref Health Africa in Kenya Ethics \& Scientific Review Committee (ESRC) and the County Government. The local leaders were also notified about the study. Ethical considerations were adhered to for participant's rights to confidentiality and privacy. Participation was voluntary and respondents were exposed to the objectives of the study, benefits, risks and inconvenience of participating prior to getting consent by those aged 16 and above. Assent was provided by those below 16 years as they were not able to provide legal consent to participate in the study. Their parents or legal guardian gave consent on their behalf. However, parental permission for married minors was not required.

\subsection{Data Analysis}

All voice-recording and verbatim transcriptions were carried out before analysis. Transcripts and data coding was done and data analyzed using thematic content analysis [23]. Transcripts were read several times by the author and an expert analyst (Co-author) to develop a coding frame using the key themes which emerged. Each transcript was coded according to the framework, with additional codes added in consultation with the author where data indicated an emerging theme. The raw data were revisited time and again, to reflect on the interpretation of the narratives. The framework was divided further into subthemes. The narratives were then recoded where relevant, under these subthemes. During coding, similar responses were organized according to the same questions in FGD, IDIs and KIIs. The coding frame for the socio-cultural factors included the following key themes: How cultural values influence daily life; opinion on what is influencing the current sexual behavior among youth; how education influences daily life; what matters the most on personal values; culture influences to ${ }^{2}$ Community health volunteers worked at the community in disseminating health information and mobilised community members during national health campaigns. They also distributed oral contraceptives and condoms. 
Table 1. Participants characteristics by region, age, gender, marital status, in-and out-of-school, occupation and religious leaders' involvement.

\begin{tabular}{|c|c|c|c|c|c|c|c|}
\hline & \multirow{2}{*}{$\begin{array}{c}\text { Age } \\
\text { (years) }\end{array}$} & \multicolumn{3}{|c|}{ Samburu } & \multicolumn{3}{|c|}{ Turkana } \\
\hline & & FGD & IDI & KII & FGD & IDI & KII \\
\hline Male in-school & $10-14$ & 2 & 2 & & 1 & 2 & \\
\hline Female in-school & $10-14$ & 2 & 2 & & 1 & 3 & \\
\hline Male in-school & $15-19$ & 2 & 1 & & 2 & 4 & \\
\hline Female in-school & $15-19$ & 2 & & & 2 & 1 & \\
\hline Male unmarried out-of-school & $15-19$ & 2 & 3 & & 3 & 3 & \\
\hline Female unmarried out-of-school & $15-19$ & 1 & 4 & & 3 & 3 & \\
\hline Male married out-of-school & $15-19$ & & 1 & & 0 & 4 & \\
\hline Female married out-of-school & $15-19$ & 2 & 1 & & 1 & 1 & \\
\hline Male married out-of-school & $20-24$ & & 2 & & 3 & 4 & \\
\hline Female married out-of-school & $20-24$ & 2 & 3 & & 2 & 3 & \\
\hline Morans* & & 3 & 2 & & & & \\
\hline $\begin{array}{l}\text { Male youth leaders religious } \\
\text { congregation }\end{array}$ & & 1 & & & 1 & 1 & \\
\hline $\begin{array}{l}\text { Female youth leaders from religious } \\
\text { congregations }\end{array}$ & & 1 & & & 1 & & \\
\hline Health Care Providers (Public) & & & & 1 & & & 6 \\
\hline Health Care Providers (Private) & & & & 2 & & & 2 \\
\hline $\mathrm{CHV}$ & & & & 5 & & & 9 \\
\hline Clinic/chemist attendants (private) & & & & 2 & & & 3 \\
\hline Male teachers & & & & 2 & & & 3 \\
\hline Female teachers & & & & 1 & & & 2 \\
\hline Male parents of the youth & & & & 1 & & & 2 \\
\hline Female parents of the youth & & & & 2 & & & 4 \\
\hline Spiritual leader & & & & 1 & & & 1 \\
\hline Activist (Traditional leaders) & & & & 1 & & & 1 \\
\hline Total & & 20 & 21 & 18 & 20 & 29 & 33 \\
\hline Urban & & 8 & 13 & 11 & 12 & 13 & 16 \\
\hline Rural & & 12 & 8 & 7 & 8 & 16 & 17 \\
\hline
\end{tabular}

${ }^{*}$ Morans are circumcised unmarried male youth whose role is to protect the community.

sexual relationships among youth; opinion on the average age of first sex encounter by girls and boys and; what role social norms play regarding sex and contraceptives in society. Verbatim quotations were used to illustrate responses [23]. Data analysis was done by use of Atlas.ti software.

The study had 2 limitations in collecting the information related to sexual reproductive health information and utilization issues. Responses were recorded by participants' age group and not by level of education. It was therefore not possible to report the myths and misconceptions by level of education. The study did not include cultural leaders from the pastoral communities. It was therefore 
not possible to report their cultural opinions on sexual reproductive health seeking behavior by adolescents.

\section{Results}

The results of this study are arranged mainly into five broad categories that is demographic characteristics, socio-cultural factors, awareness and knowledge, myths and misconceptions as well as fears of family and community. The findings are thereafter organized into three categories of demographics, barriers and enablers to utilization of SRH services and information.

\subsection{Demographic Characteristics}

Table 1 gives a summary of characteristics of participants' based on region, age, in-school and out-of-school ${ }^{3}$, gender, marital status among other factors considered for data collection.

\subsection{Barriers}

Socio-cultural factors. The study revealed that cultural practices in both Samburu and Turkana pastoral communities influenced utilization of SRH services. In these communities, early marriage was encouraged as a source of income for the family and as a prestigious way of getting wealth. The community believed that having children was a blessing from God. This was noted in most of the discussions as presented below:

As youths we undergo a lot of problems, some parents force girls to marry so as to get money... Others make their children drop out of school to do casual jobs so as to cater for family needs (FGD, 15 - 19 yrs. female only out-of-school group, Samburu County).

According to the teachers, it was noted that girl's engagement in early sexual activities is a socio-cultural issue which is encouraged by their parents:

My experience in this place is that girls engage in sex when very young... they engage in sex as early as ten years. I receive pregnancy complaints in my school every year and this has made me worried since they drop out of school to get married... the fertility of girls here is very high. I have shared the complaints and my concern with (ward) administrator to find a solution to this problem (KII, male teacher, Turkana County).

This was also supported by some of the adolescents who took part in the study who confirmed that cultural issues influence sexual behavior as it encourages early marriages:

"...having children is seen as a blessing from God to the community..." (IDI, female out-of-school, Turkana County).

The study revealed that women do not have a voice in the presence of men and are considered "children" if not circumcised. It is believed that such (uncircumcised) women could not be trusted to make decisions including on their re- 
productive health. Girls are also taught not to say no to sexual advances from men and are encouraged to have sexual relationship. This information was captured during in-depth interviews:

Women do not have a voice in front of men and even worse if uncircumcised. Women who are not circumcised are still considered to be children (IDI, Female 20 - 24 years married, Samburu County).

Girls are taught not to say no and will often not turn down men who want to have sexual relationship with them (IDI, Female out of school 15 - 19 years unmarried, Samburu, County).

In Turkana girls are forced to marry early and their parents result to organizing for rape if a daughter refuses the suitor selected for her. The rape is mostly organized if the suitor is wealthy. The parents organize the suitor's friends to rape the daughter and once raped the girl is pronounced married:

Our culture determines when a girl should be married. If a daughter does not want to get married, parents organize young men who are the suitor's friends to make sure that they rape her and therefore she is forced to accept the man (KII, Health care Provider, Turkana County).

In Samburu, women are encouraged to have children out of wedlock. The community believed that if you bore children only with your husband and he was a coward, your Morans might be killed in war. It was therefore advisable to have children with different brave men. Despite the risk of contracting sexual transmitted disease as a result of multiple sexual partners, Morans didn't go to hospitals as they preferred to go to herbalists as indicated in female parent KII:

Our culture allows women to have children out of wedlock. If you bore children with only your husband and he was a coward, your Moran's might be killed in war, so it's advisable to have children with different men who are brave (KII, Female parent, Samburu County).

In Turkana the community believes a married woman should be protected from sexual disrespect. An animal is slaughtered and blood smeared on her body and no one is allowed to tough her body to prevent her from going outside of marriage as indicated in the male FGD:

There are cultural practices that married women are expected to follow. An animal is slaughtered and a woman is smeared with a red stuff (like clay) to prevent her from going outside of marriage. She is not supposed to allow people to touch her body or her house things. This protects women from contracting sexually transmitted diseases (FGD, male in school, 10 - 14 years, Turkana County).

Samburu community practiced female genital mutilation (FGM), which is mostly done between the ages of 10 - 14 years. After circumcision, the adolescents are considered mature and therefore allowed to engage in sex or marriage. The information was captured during male FGD discussions:

After circumcision for both boys and girls, you are adults and can engage with what adults do (sex). 
(FGD, male out-of-school, 15 - 19 years, Samburu County).

Myths and misconceptions. The study results showed that myths and misconceptions acted as individual barriers towards sexual reproductive health utilization among the youth. The main misconceptions mentioned were that family planning causes infertility while condoms caused cancer as mentioned in male KII:

Family planning makes a woman unable to conceive and if she conceives she will not give birth to a healthy baby. Some people also say that condom brings cancer (KII, male activist, Samburu County).

Participants in the study believed that implants can get lost in the body while family planning makes girls to become prostitutes and also causes weight gain. This was captured during the female FGD group:

Youth are discouraged from the experiences of their friends who have received family planning injections and have gained weight. Girls who use family planning become prostitutes because they have nothing to fear. Youth also fear that 5 year implants can be lost in the body and also cause infertility (FGD, out-of-school, female unmarried, 15 - 19 years, Samburu County).

Some of the youth especially Moran trusted that herbal drugs were much better in the cure of sexually transmitted infections and they would therefore not go to the hospitals for treatment as discussed in the female FGD group: ( $F G D$, female out-of-school, 15 - 19 years, married, Samburu County).

Morans do not seek medical treatment but prefer herbalist for treatment of sexual transmitted diseases (FGD, out-of-school, 15 - 19 years, unmarried, Samburu County).

Some of the parents of the youth were concerned about youth practice towards unprotected sex. This was captured from a male FGD:

Some of the youth want to have an experience of having sex without protection. They are not using condoms because they are still young and they want to have that feeling of having sex without protection (FGD, out-of-school, 15 - 19 years, unmarried, Turkana County).

Fear of the family and the community by youth seeking SRH services and information from the health facilities: The study results show that the community had an influence on adolescents and youth who went to the health facility for SRH health services. The youth feared to be seen at the health facilities. Further, custom disallowed girls to talk to older people and this hindered them from visiting the clinics. Youth also feared that a health care provider would inform their parents if found to have a positive result of a disease. It was shameful for a youth to visit a health facility. The youth also feared to be seen taking pills because the community would think that the girl was taking pills because she had HIV/AIDS and therefore be stigmatized. This was reported in the male FGD group:

It is true that the youth fear their family members who might cause a confrontation by their parents. Youth also fear that if a health provider is not confidential she might disclose test results to many people and that might cause stig- 
matization in the community (FGD, male, in-school, 10 - 14 years, Samburu County).

The Morans feared to be seen talking to a lady at the health facility. Further, men were not expected to visit the clinics. This was captured from the KII participant:

Being a Moran, we fear to be seen by other people while visiting the hospital because Morans are not supposed to interact with women. Youth also fear going to the health facility because of traditional custom. For example, girls won't talk to older people and this prevents them from going to the clinic (KII, CHV, Samburu County).

\subsection{Enablers}

Urbanization, education and technology: Although culture in both communities of Samburu and Turkana influenced SRH utilization the study showed evidence of changes with potential to enhance utilization of SRH information and services. These changes included urbanization, education and technology.

Urbanization was influencing the culture as more people were aware of the importance of education and modernization. In urban areas, parents were taking their children to school, preventing early marriages and did not live in manyattas (local traditional houses). The value of early marriage was changing, and increasingly more parents wanted their children to complete their education first before getting involved in sexual behaviors. Parents believed that education would improve their children's socio economic status. The concerns of the parents were captured from the male KII:

Every youth should make sure that they finish their education first before getting involved in sexual behavior because it is good for one to get a place to live before getting married. The parents should also not neglect their children. They should counsel them about the importance of education and sexuality issues so that they do not mess with their lives (KII, male parent, Turkana County).

With education and exposure to information more youth were getting aware of dangers of unprotected sex and health services offered at health facilities. Youth participating in the study believed that health is important and SRH services protect them from diseases including HIV/AIDS as indicated through FGDs for male congregants and Morans.

The value of health is changing. Health is very important in that if you are not healthy, you can't cater for your needs in life. If you are not healthy also, people will look down upon you especially us the youths. We are living in dangerous times where we must be extra careful in the way we live. Diseases like HIV/AIDS are all over so we must maintain and protect ourselves (FGD, male congregant, Samburu County).

...youth believe that condoms help us to prevent sexually transmitted diseases (FGD, Morans, unmarried 20 - 24 year, Samburu County).

Health care providers believe that current youth especially in towns increasingly use family planning and visit clinics more as indicated in the health care 
provider KII discussion:

Today, youth are more enlightened compared to our times. They also use family planning more than at our time (KII, health care provider, Samburu County)

The study shows evidence of improved communication on SRH issues through modern technology. Increasingly, more youth owned mobile phones and were able to communicate to each other on where to get services as indicated in the male out of school IDI:

We now get SRH information through the local radio. We also have phones to communicate with friends and also download information on contraceptives and HIV. My phone has WhatsApp, face book and proper Internet connection (IDI, male, out-of-school, 20 - 24 years, married, Turkana, County).

Further, more families in urban centers owned television sets and therefore people including the youth listened to topics that discussed reproductive health issues. The following was captured in the female FGD youth:

We use television, radio and Internet to get information. We also get information from magazines and newspapers. Common ways we communicate through are blogs, websites, Facebook, WhatsApp and phones through SMS' (FGD, female, religious congregant, Samburu County).

There are SRH programs in the community that have influence on cultural practices. The study revealed that some of the NGOs are training youth on the effects of early marriage and FGM on health as well as the importance of education. This information was captured from a female IDI:

Youth are now seen as the leaders of tomorrow and that is why they are in school. There are also some NGOs who come to teach youth on alternative rites of passage, while discussing early marriages and FGM (IDI, female, 10 - 15 years in school, Samburu County).

Awareness and knowledge: The culture in both communities of Samburu and Turkana values children as a blessing to the community and does not include education or counseling on sexual protection to prevent infections. Youth also believe that if they are taught how to use RH services then they would use them.

The participants in the study mentioned their needs to include awareness and knowledge of contraceptives and other SRH service as discussed in the female FGD group:

As youth we fear using condoms because we do not have the knowledge on how they should be used... if I am taught how to use these services, then I will use... Youth should also be given advice on sexual reproduction heath issues and counseled on how to use them (IDI, female, 10 - 15 years in school, Samburu County).

Among the SRH needs mentioned were desire to know how contraceptives work, information about reproductive health and where to get SRH services near where they lived. This was reported in male FGDs:

There are those who live very far away (remote areas) or go to graze cattle 
very far from the health centers. They lack information about the source, availability and the importance of contraceptives (FGD, male, out-of school, 15 - 19 years, Turkana County).

\section{Discussion}

The SRH ill-health continues to mostly affect adolescents and youth worldwide [1]. The poor RH outcomes are as a result of inadequate use of SRH services [2].

This study attempted to identify some of the critical factors that influenced utilization of SRH services and information in the two Counties of Samburu and Turkana in Kenya. The study findings would contribute towards developing appropriate strategies to increase utilization of SRH services among the youth. Both pastoral communities had similar practices in their ways of life for example early marriage and value of children. However, unlike Samburu, Turkana people do not practice female genital mutilation (FGM).

Among the key factors found to influence SRH information and services utilization included socio-cultural factors. Socio-cultural factors influenced formation of myths and misconceptions on contraceptives and fear of family and community by the youth seeking SRH services from the health facilities. This finding is consistent with other studies. According to Hulton et al. (2000), socio-cultural factors influence how decisions are made and the confidence to use SRH services or not to use [24].

Children were seen as a blessing to the community and parents encouraged early marriage. This finding supports the value expectancy model by Janz and Becker that considers the costs and benefits associated with engaging in or avoiding a specific type of behavior [25]. Literature also shows that the influence of the family or community support or responsiveness on adolescent behavior represent the central ways in which parents influence healthy adolescent development [11]. In addition, marriage for many girls is the beginning of frequent and unprotected sexual activity. Studies conducted elsewhere show that early and unprotected sexual activities expose young people to pregnancy-related health complications and sexually transmitted infections including HIV/AIDS [3]. Strategies developed to address early marriage, spacing of births, girl child education and disadvantages of FGM would be important in reducing TFR and sexually transmitted diseases.

Results show that women were allowed to have children with different men, and girls were not allowed to say no to men who approached them for sex while adolescents were considered mature to engage in sex after circumcision which was carried out between $10-14$ years. This result conforms with the theory of reasoned action behavior- that behavior is influenced by various factors among them one's assessment that a particular behavior is desired by significant others and a motivation to comply with the view of other people in the environment who would be in this case, family and peers [8] [24] [26]. Sexuality information should be examined in schools to ensure that both males and females have information on gender violence and the community educated on the protection of 
girls against forced sex and its consequences on the health of the youth.

Results showed that women did not have a voice in the presence of men and could not be trusted to make decisions at home including on their own sexuality. It would therefore be difficult for a woman to seek SRH services without the approval of the partner. A study by Gage shows that sexual partner communication increases contraceptive use [27]. In a study carried out in Kenya, sexual partner communication significantly influenced contraceptive use among adolescents [28]. There is need to develop behavior change communication materials that encourage partner communication on SRH issues for the community.

In this study, urbanization was exposing youth to SRH information and services through education and technology. Parents were encouraging their children to complete the education before marriage. From the narratives, more youth were using contraceptives compared to their parents. According to Kenya Demographic Health Survey of 2014, contraceptive use is associated with higher levels of education [4]. In another study, adolescents living in urban and semi-urban areas had access to mass media and had desired influence on risk perception on contraceptive use [29].

One of the key needs mentioned by adolescents and youth was to know how condoms are used. Studies show that contraceptive use increases with the level of knowledge of how they are used [29] [30]. A study in Kenya found that use of contraception was significantly higher among adolescents 15 - 19 years who knew how to use contraceptives than those who did not know [28].

Being adolescent affects perception of sexuality and was found significant in this study where some of them indicated they wanted to have sex without condoms when still young. Myths and misconceptions influenced utilization of SRH services. Youth trusted herbal drugs more than conventional drugs in the treatment of sexually transmitted diseases. Further, the youth believed in having unprotected sex when they were still young. These findings are consistent with theory of perception formation on barriers to contraceptive use [30] [31]. These myths influence contraceptives use and expose youth to unprotected sex hence risk to sexually transmitted diseases including unwanted pregnancies. Accurate information on sexuality would enhance desired perceptions and informed decision on contraception by adolescents and youth.

Low esteem among some youth has been associated with non-use of contraceptives and other SRH information and services [32] [33]. The results show that youth feared their relatives and friends who discouraged them from visiting health facilities. Youth also feared stigmatization if the health provider does not observe confidentiality of their test results. Further, culture does not allow girls to talk to older people while Morans are not allowed to talk to women and therefore this expectation limited the number of youth seeking SRH information and services from health facilities. Similar findings are reported elsewhere for example, in Bolivia, adolescents feared being punished by family and ridiculed by peers and this negatively affected their ability to seek services [16]. Majority of health workers are women and if young people are afraid to talk to women or 
older people, this would have health implications on those of them who get pregnant and will not attend antenatal care or seek treatment for sexually transmitted infections. There is need therefore to develop culture specific strategies on the importance of $\mathrm{RH}$ information and utilization of services by the youth integrating the gate keepers in the community that includes teachers, parents, leaders and youth. Parents should also be involved in the development of community specific messages for parental education on SRH issues. Parental involvement in the planning and execution of youth program and the formation of parental support programs is critical if youth programs are to succeed in increasing utilization of SRH services and information.

\section{Conclusion}

To enhance adolescents' and youth's utilization of SRH services, it is important to educate them and build their confidence in the decision-making process through focusing on messages that give information on how contraceptives are used and where to get them. Adolescents are engaging in sex as early as 10 years. Some adolescents wanted to experience sex without use of condoms. This finding has implications on school dropouts. Hence, there is the need to build capacity of schools to educate their students on sexuality and reproductive health issues and the importance of seeking treatment for sexually transmitted infections including HIV testing from health facilities. Health care providers should work with parents, community leaders and teachers to strengthen their knowledge in understanding of the adolescence stage, challenges, needs in preventing unsafe sex, and prevention of unwanted pregnancy, sexually transmitted infections and disadvantages of FGM including early marriage. Adolescents should also be encouraged to discuss contraceptive use with their sexual partners. To effectively address utilization of SRH services in the community, efforts should be made to involve the community members to support organizing mobile clinics in the pastoral areas to offer SRH services and information to young people living far from health facilities. Further research should also be carried out to investigate activities that can attract youth to health facilities for SRH services and information.

\section{Acknowledgements}

This study was made possible by the support of the American People through the United States Agency for International Development (USAID.) The contents of the study are the sole responsibility of Afya Timiza Project and do not necessarily reflect the views of USAID or the United States Government.

\section{Conflicts of Interest}

The authors declare that they have no competing interest.

\section{References}

[1] Haile, S.A. (1995) International Conference on Population and Development, Cairo 
5-13 September 1994, IAC Presence. Newsletter (Inter-African Committee on Traditional Practices Affecting the Health of Women and Children), 17, 19.

[2] Magadi, M. (2006) Poor Pregnancy Outcomes among Adolescents in South Nyanza Region of Kenya. African Journal of Reproductive Health, 10, 26-38. https://doi.org/10.2307/30032441

[3] United Nations Population Fund. (2001) Population Issues: Briefing Kit. 9-17. https://shop.un.org/books/population-issues-brfing-kit-2001-24340

[4] (2015) National Bureau of Statistics-Kenya and ICF International, 2014 KDHS "Key Findings”. KNBS and ICF International, Rockville, Maryland, USA.

[5] Kenya National Bureau of Statistics (KNBS). (2009) Population and Housing Census. Republic of Kenya, Nairobi.

[6] Ministry of Health (MOH), Kenya. (2015) National Adolescent Sexual and Reproductive Health Policy. MOH.

[7] Glanz, K., Rimer, B.K. and Lewis, F.M. (2002) Concepts of the Social Cognitive Theory Health Behaviour and Health Education: Theory, Research and Practice. Wiley and Sons, San Francisco, 176-177.

[8] Bandura, A. (1986) Social Foundations of Thought and Action: A Social-Cognitive Theory. Prentice-Hall. Inc., Englewood Cliffs.

[9] Hulton, L.A., Rachael, C. and Symons, W.K. (2000) Perceptions of the Risk of Sexual Activity and Their Consequences among Ugandan Adolescents. Studies in Family Planning, No. 31, 35-46.

[10] Meekers, D. and Klein, M. (2002) Determinants of Condom Use among Young People in Urban Cameroon. Studies in Family Planning, 33, 335-346. https://doi.org/10.1111/j.1728-4465.2002.00335.x

[11] Simpson, A.R. (2001) Raising Teens: A Synthesis of Research and a Foundation for Action; Project on the Parenting of Adolescents. Center for Health Communication, Harvard School of Public Health.

[12] Kinaro, J., Kimani, M., Ikamari, L. and Ayiemba, E.H.O. (2015) Perceptions and Barriers to Contraceptive Use among Adolescents Aged 15 - 19 Years in Kenya: A Case Study of Nairobi. Health, 7, 85-97. https://doi.org/10.4236/health.2015.71010

[13] Godia, P.M., Olenja, J.M., Hoffman, J.J. and Brook, N. (2014) Young People's Perceptions of Sexual and Reproductive Health Services in Kenya. BMC Health Services Research, 14, 172. https://doi.org/10.1186/1472-6963-14-172

[14] Derrel, W. (2000) Selective Perception. http://www.ciadvertizing.org

[15] Harper, C., et al. (2004) Adolescent Clinic Visits for Contraception: Support from Mothers, Male Partners and Friends. Perspectives on Sexual and Reproductive Health, 36, 20-26.

[16] de Belmonte, L.R., Gutierrez, E.Z., Magnani, R. and Lipovsek, L. (2000) Barriers to Adolescents' Use of Reproductive Health Services in Three Bolivian Cities, Washington DC. Focus on Young Adults/Pathfinder International. https://www.semanticscholar.org

[17] Agha, S. (1998) Sexual Activity and Condom Use in Lusaka, Zambia. International Family Planning Perspectives, 24, 32-37. https://doi.org/10.2307/2991917

[18] Ghosh, J., Kalipeni, E. and Malawi, E. (2005) Women in Chinsapo, Malawi: vulnerability and risk to HIV/AIDS. Journal of Social Aspects of HIVI AIDS, 2, 320-332. https://www.tandfonline.com/doi/pdf/10.1080/17290376.2005.9724857

[19] UNAIDS, WHO (2008) Report on the Global AIDS Epidemic. UNAIDS, New York. 
http://www.unaids.org/sites/default/files/media_asset/jc1510_2008globalreport_en_ 0.pdf

[20] Dlamini, B.R., Mabuza, P., Thwala-Tembe, M., Masangane, Z., Dlamini, P. and Simelane, E. (2017) Are Adolescents and Youth Programs Missing the Real Targets? Analysis of Socio-Cultural Factors Influencing Use of Sexual Reproductive Health Services by Young People in Swaziland. Journal of AIDS and Clinical Research, No. 8,684 .

[21] Kirby, D. (2002) The Impact of Schools and School Programs upon Adolescent Sexual Behavior. Journal of Sex Research, 39, 27-33. https://doi.org/10.1080/00224490209552116

[22] Kenya National Bureau of Statistics (KNBS). (2010) About Samburu County in Kenya 2009 House Census: Explore by County. http://www.kenya-information-guide.com/samburu-county.html

[23] Burnard, P. (1991) A Method of Analyzing Interview Transcripts in Qualitative Research. Nurse Education Today, 6, 461-466. https://doi.org/10.1016/0260-6917(91)90009-Y

[24] Hulton, A.L., Cullen, R. and Khalokho, S.W. (2000) Perceptions of the Risk of Sexual Activity and Their Consequences among Ugandan Adolescents. Studies in Family Planning, 31, 35-46. https://doi.org/10.1111/j.1728-4465.2000.00035.x

[25] Nancy, K., Janz, R.N. and Marshal, H.B. (1984) The Health Brief Model. A Decade Later. Health Education Quarterly, 11, 1-47.

[26] Bandura, A. (2001) Social Cognitive Theory: An Agentic Perspective. Annual Review of Psychology, 52, 1-26. https://doi.org/10.1146/annurev.psych.52.1.1

[27] Gage, A.J. (1998) Sexual Activity and Contraceptive Use: The Components of the Decision Making Process. Studies in Family Planning, 29, 154-166.

https://www.ncbi.nlm.nih.gov/pubmed/9664629 https://doi.org/10.2307/172156

[28] Kinaro, J.W. (2015) Contraceptive Use among Adolescents: Perceptions and Barriers. Lap Lambert Academic Publishing, Saarbrücken, 146-172.

[29] Haque, M.R. and Soontharndhada (2009) Risk Perception and Condom Use among Thai Youths: Findings from Kanchanaburi Demographic Surveillance Systems Site in Thailand. Journal of Health Population and Nutrition, 27, 772-783.

[30] Kinaro, J.W. (2013) They Will Wonder What Kind of a Girl I Am: Adolescent Perceptions towards Contraceptive Use in Nairobi. Advances in Sexual Medicine, 3, 1-10. https://doi.org/10.4236/asm.2013.31001

[31] Wingood, G., Ralph, J., Harrington, K., Edward, W. and Kim, M. (2001) Exposure to X-Rated Movies and Adolescents' Sexual and Contraceptive-Related Attitudes and Behaviors. Pediatrics, 107, 1116-1119. https://doi.org/10.1542/peds.107.5.1116

[32] Erulkar, A.S., Ettyang, L.I., Onoka, C., Nyagah, F.K. and Muyonga, A. (2004) Behavior Change Evaluation of a Culturally Consistent Reproductive Health Programme for Young Kenyans. International Family Planning Perspectives, 30, 58-67. https://www.ncbi.nlm.nih.gov/pubmed/15210404 https://doi.org/10.1363/3005804

[33] Erulkar, A.S., Onoka, C.J. and Phiri, A. (2005) What Is Youth Friendly Adolescent's Preferences for RH Services in Kenya and Zimbabwe. Africa Journal of Reproductive Health, 9, 51-58. http://www.popcouncil.org

https://doi.org/10.2307/3583411 